\title{
El teletrabajo: nueva modalidad laboral y una opción digital para las empresas y la sociedad
}

\author{
Fecha de recepción: 15/10/2019 • Fecha de aceptación: 09/12/2019 • Fecha de ublicación:10/02/2020
}

Juan Carlos Vargas Espín

Servicio de Gestión Inmobiliaria del Sector Público - INMOBILIAR

jcvargas001@gmail.com

https://orcid.org/0000-0003-1258-1167

\section{RESUMEN}

Las tendencias globales apuntan a la transformación de los entornos laborales, entre estas aparece una nueva modalidad laboral apoyada en las Tecnologías de la Información y las Comunicaciones denominada Teletrabajo, misma que combina políticas corporativas basadas en cumplimiento de objetivos. El Gobierno del Ecuador a través del Ministerio de Telecomunicaciones y de la Sociedad de la Información ha impulsado como proyecto la implementación de Infocentros Comunitarios a nivel nacional, mismos que constituyen espacios tecnológicos de participación y desarrollo que garantizan el acceso inclusivo a las Tecnologías de la Información y Comunicaciones de las poblaciones de parroquias rurales y urbanos marginales. Estos espacios permiten ampliar los emprendimientos, donde la economía plantea el desarrollo a través de la transformación digital de las organizaciones, la evolución del comercio electrónico, el impulso de la innovación y emprendimientos de base tecnológica, que han permitido que las TIC encaminen al teletrabajo como una nueva alternativa laboral, donde las fuentes de empleo no se encuentren atadas a la presencia física del empleado, impulsando de manera incluyente el desarrollo de habilidades digitales en los usuarios a través de herramientas y aplicaciones tecnológicas que mejoren la calidad de vida del ciudadano e impulsando el desarrollo productivo de su comunidad.

PALABRAS CLAVE: teletrabajo, infocentros, MINTEL, TIC, empleo. 


\begin{abstract}
The global trends point to the transformation of the working environments, among these appears a new working modality supported by the Information and Communication Technologies called Telework, which combines corporate policies based on the fulfillment of objectives. The Government of Ecuador, through the Ministry of Telecommunications and the Information Society, has promoted the implementation of Community Infocenters at the national level, which are technological spaces for participation and development that guarantee inclusive access to information and communication technologies for the populations of rural and marginal urban parishes. These spaces allow the expansion of enterprises, where the economy proposes development through the digital transformation of organizations, the evolution of electronic commerce, the promotion of innovation and technology-based enterprises, which have allowed ICT to lead to teleworking as a new employment alternative, where the sources of employment are not tied to the physical presence of the employee, promoting in an inclusive manner the development of digital skills in users through technological tools and applications that improve the quality of life of the community. to the physical presence of the employee. Furthermore, it promotes the development of digital skills through tools and technological applications that improve the quality of life of the citizens and enhances the productive development in their community.
\end{abstract}

KEYWORDS: teleworking, infocentros, MINTEL, TIC, employment 


\section{INTRODUCCIÓN}

En el año 1973, Jack Nilles lidera a un grupo de científicos estadounidenses de la Universidad de California del Sur, en la realización de estudios sobre la aplicación de las tecnologías informáticas para rentabilizar los beneficios económicos como respuesta a la crisis del petróleo emergida en ese entonces. El trabajo de Nilles planteaba reducir costos asociados al uso de combustible vinculados al desplazamiento de los trabajadores en las horas pico y a su vez generar una reducción de emisiones gaseosas nocivas para el medio ambiente. En resumen, se buscaba evitar el traslado físico del trabajador más no de la información necesaria para la empresa, acción que podía realizarse desde su domicilio o el centro de trabajo más próximo. Gracias a esta investigación, se le otorgó a Nilles el seudónimo de "padre del Teletrabajo" (Ushakova, 2015).

El teletrabajo, nacido de la investigación científica, ha sufrido e incorporado variantes a lo largo de las últimas décadas alentado sobre todo por el desarrollo que ha sufrido la tecnología y la implantación de Internet. Esto la convierte en una forma de trabajo eficaz, con menores costos fijos y mayor flexibilidad en la adaptación de las empresas a las necesidades del mercado (Civit \& March, 2000). Para muchas personas y empleadores, el Teletrabajo no es más que una nueva cara del trabajo tradicional en el domicilio, observación por demás válida.

Sin embargo, es importante diferenciar los términos trabajo remoto versus trabajo a domicilio, siendo que el teletrabajador no necesariamente acceda a esta modalidad laboral desde su casa, más aún si no dispone de herramientas basadas en Tecnologías de la Información y Comunicación (TIC). El trabajo remoto denominado teletrabajo genera una relación contractual formal entre el empleado y empleador (Ministerio Relaciones Laborales, 2018). Al expresarlo de forma práctica como fórmula sería: Teletrabajo = Trabajo + Distancia + Uso intensivo de las TIC, en donde; sus tres elementos son claves para su óptima aplicación (Havriluk, 2010).

El mercado laboral mundial ha venido registrando un crecimiento acelerado, donde las tecnologías de la información y comunicaciones representan el medio por el cual las organizaciones y empresas transnacionales generan sus actividades de manera cotidiana, por lo que aproximadamente un $20 \%$ de la población mundial accede al teletrabajo como modalidad laboral, considerando que su conexión remota la realizan al menos una vez por semana.

Los teletrabajadores optimizan su tiempo sumado a la capacidad de trabajar de forma remota y en horarios flexibles, donde las TIC se convierte en la herramienta de primera línea para ejecutar sus actividades, obteniendo de esta manera una mejor calidad de vida. Sin embargo, el uso del teletrabajo en Asia, Europa y Estados Unidos es mucho más extendido. El informe ConsumerLab Insight Summary Report de Ericsson (2015), muestra que, para los teletrabajadores ubicados en estas latitudes, a sus motivaciones económicas hay que sumarle un crecimiento profesional.

Latinoamérica constituye la región con una tendencia en crecimiento en Teletrabajo, donde la principal fuerza laboral son las personas jóvenes comprendidas entre 20 a 35 años de edad, fruto de las altas tasas de crecimiento latinoamericanas, denominada Generación "Y" o "Millennials", donde; se pretende disponer de flexibilidad laboral en horarios no definidos, con la finalidad de encontrar estabilidad y balance en la vida cotidiana. Actualmente existen empresas 
transnacionales que aplican la modalidad de teletrabajo como política laboral, tal es el caso de las empresas HP, Cisco, IBM, entre otras, donde los teletrabajadores acceden a plataformas digitales corporativas de administración y generan soporte, configuración y administración de manera remota.

De acuerdo a la referencia situacional del teletrabajo a nivel mundial y en América Latina, Ecuador empieza un avance significativo en esta materia desde el año 2016. La investigación académica en el tema Teletrabajo no es ajena a esta tendencia, y en comparación con algunos países y regiones del mundo, el Ecuador ha tenido una muy baja tasa de generación de artículos en revistas indexadas en esta materia, alcanzando un $2 \%$ (Bonilla, Plaza, Soacha, \& Riaño, 2014).

Finalmente, haciendo uso de la herramienta online Google Trends, que permite visualizar la tendencia y popularidad de búsqueda de palabras en la red, el término "Teletrabajo" arroja los siguientes resultados de popularidad en los últimos cinco años (Jul 2013-Jul 2018):

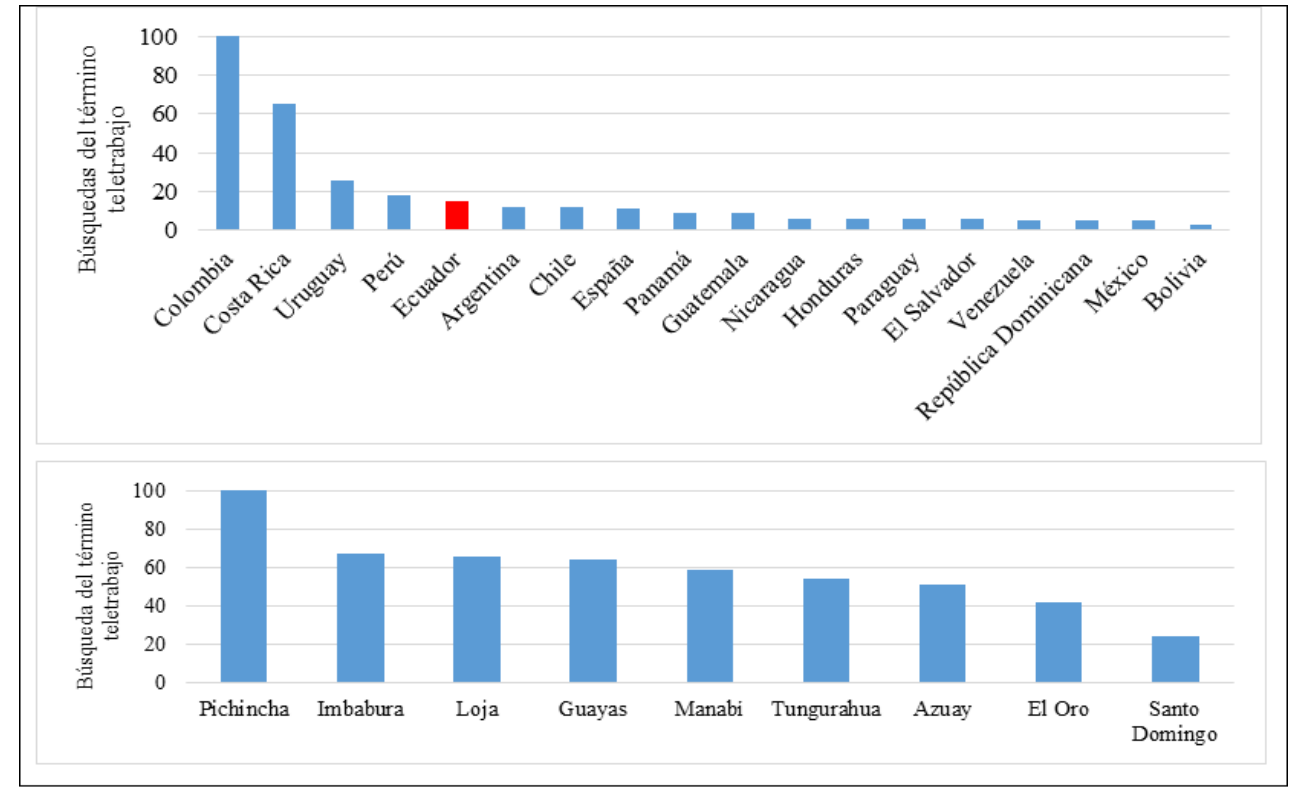

Figura 1. Pupularidad de búsqueda. Generado por Google Trends

La Figura 1 demuestra que Ecuador aparece en el grupo de países en los cuáles el término Teletrabajo aún no es muy popular o extendido en la población con acceso a TIC, siendo las provincias de Pichincha e Imbabura las que encabezan su interés.

Mediante Acuerdo No. MDT-2016-0190 emitido el 24 de agosto de 2016, el Ministerio del Trabajo establece la normativa para regular el teletrabajo, donde entre sus puntos principales se destaca que la modalidad de Teletrabajo es voluntaria y se acuerda entre las partes. Además, los teletrabajadores tendrán los mismos derechos y obligaciones que aquellos empleados que trabajen permanentemente en la empresa (Ministerio del Trabajo, 2016).

La modalidad de teletrabajo busca incrementar la productividad de las organizaciones públicas 
y privadas, con mayor inclusión de personas con capacidades especiales, disminución de tiempos y distancias, a fin de generar una movilidad sostenible con una mejora en la calidad de vida de los trabajadores. Al ser una modalidad laboral nueva en el mercado ecuatoriano, es necesario que la cultura de los teletrabajadores vaya madurando, a esto debe sumarse las políticas gubernamentales e institucionales para brindar las facilidades a los ciudadanos que accedan al teletrabajo, sin embargo; como se describió anteriormente, para poder llevarlo a cabo un teletrabajador requiere del conocimiento y uso intensivo de las TIC en el desarrollo de sus actividades. Desafortunadamente, este acceso no se encuentra ampliamente extendido en el país y zonas o parroquias rurales o marginales no cuentan con un acceso igualitario.

El Gobierno del Ecuador a través del Ministerio de Telecomunicaciones y de la Sociedad de la Información implementa espacios comunitarios de participación y desarrollo denominados Infocentros Comunitarios, donde se garantiza a la ciudadanía de parroquias rurales y urbanas marginales del Ecuador, el acceso inclusivo a las TIC, a fin de reducir la brecha de acceso y analfabetismo digital.

Los Infocentros Comunitarios ofrecen acceso a la infraestructura tecnológica implementada de manera gratuita a los ciudadanos, con la disponibilidad de herramientas tecnológicas y capacitaciones sobre el manejo adecuado TIC, uso de los servicios electrónicos gubernamentales y emprendimientos que en conjunto conllevan al mejoramiento de la calidad de vida de la población. La modalidad de teletrabajo surge como un recurso que proporciona un valor agregado a la infraestructura tecnológica presente en los Infocentros, mismos que han sido destinados a capacitar a los ciudadanos que no cuenten con dispositivos tecnológicos en espacios comunitarios de participación y encuentro.

Por otro lado, el Teletrabajo se ha convertido en una opción más de empleo, por ello se ha visto la necesidad de buscar aplicaciones web que faciliten el acceso y búsqueda de nuevas alternativas laborales. "TELEJOB ECUADOR" es una aplicación prototipo que nace como resultado de la investigación de la modalidad laboral de teletrabajo, donde tiene como finalidad implementar una aplicación web que sirva de conexión entre las personas y los empleos disponibles, en la cual los ciudadanos pueden registrarse en el sistema, acceder a las ofertas de trabajo y postularse a las tareas que sean de su elección, a fin de contribuir en el ámbito social, cultural y económico de las personas que buscan nuevos emprendimientos y fuentes de empleos.

\section{MÉTODOS Y MATERIALES}

El tipo de investigación se realizó considerando la finalidad que persigue este estudio como es desarrollar una propuesta de marco de referencia para Teletrabajo en Ecuador, caso Infocentros del Ministerio de Telecomunicaciones y Sociedad de la Información. Se corresponde con el de una investigación de carácter explicativa, ya que en base a los hechos pretende indagar las posibles causas para construir los argumentos del marco de referencia del teletrabajo. El tema del teletrabajo es reciente en el país y existe poca documentación al respecto, por tanto, la investigación también es exploratoria ya que las fuentes de información son escasas e indirectas sobre el tema (Domínguez Garrido, 2009). 
Finalmente debido a que la investigación pretende caracterizar lo mejor posible al teletrabajo como fuente de ingreso familiar mediante la tecnología de los Infocentros Comunitarios del MINTEL, entonces la investigación posee adicionalmente un carácter descriptivo. Hurtado de Barrera (2012) asegura que este tipo de investigación "se asocia al diagnóstico, siendo el propósito exponer el evento estudiado, haciendo una enumeración detallada de sus características con la finalidad de hacer una clasificación de las mismas o ponerlas en relación para hacer una descripción más detallada".

Para el presente caso de estudio en lo concerniente al Teletrabajo en los Infocentros Comunitarios, el diseño de estudio es de campo, puesto que la aplicación del instrumento para recabar la información pertinente se realizó en in situ.

Los datos se tomaron en un solo momento para su posterior tabulación, análisis y evaluación sin repetir el proceso, por tanto, el estudio tiene un diseño experimental que permite elegir al grupo al cual será indagada la información. La población a investigar se escogió dependiendo del objetivo del estudio, siempre considerando que se lo realiza para beneficio de los ciudadanos de las comunidades rurales y urbanas marginales del Ecuador que cuentan con Infocentros.

Por otra parte, tomando como referencia las metodologías de Scharager \& Reyes (2001) y Parra (2011), se utilizó una toma y muestreo no probabilístico por situaciones de conveniencia, facilidad de acceso y porque el estudio fue dirigido a una población y grupo específico que pertenecen a las personas asistentes a los Infocentros. Se consideró una muestra no probabilística de tres (3) sujetos de estudio, los cuales son funcionarios públicos encargados de Administrar Proyectos y Gestiones Tecnológicas dirigidas principalmente a los Infocentros Comunitarios del MINTEL. Los funcionarios reconocen el avance obtenido hasta el momento en materia de Tecnologías de la Información y Comunicaciones permitiendo una reducción en la brecha digital y generando un alto crecimiento tanto en el sector público como privado a pesar de los problemas económicos que han impactado al Ecuador.

Después de establecer claramente los procesos metodológicos para la respectiva investigación se procedió a elegir de manera adecuada los instrumentos que van a intervenir para la recolectar la información. La investigación no tiene sentido sin las técnicas de recolección de datos, las mismas que conducen a la verificación del problema planteado. Para la recolección de datos se escogió la técnica de metodología cualitativa que es la entrevista, en ella se realizan un par de preguntas a un grupo de personas seleccionas que sepan de la situación del tema de estudio. Su fortaleza radica en que una persona involucrada es el sujeto idóneo para detallar y ahondar en la experiencia generada. Se procuró recolectar todo este bagaje experimental en la creación de este marco metodológico dirigido a los Infocentros Comunitarios.

Una vez que se han obtenido los datos es necesario realizar su análisis a fin de descubrir lo que significa en función a los objetivos planteados para la investigación, por tanto, es necesario escoger el tipo de análisis apropiado. Sobre este punto, Behar (2008) afirma que en sus investigaciones la manera más correcta y adecuada para dar un sentido a la información recogida al inicio del proceso de investigación es diseñar estadísticas de las aptitudes y opiniones que ayuden a ver qué criterios son buenos y cuáles no, pero de ellas las positivas permiten identificar 
que es un impacto fundamental en la sociedad como es la modalidad del teletrabajo que es un medio por el cual las personas trabajan desde casa y tiene varias comodidades y facilidades, mientras que con las negativas que sean identificadas se realizar un análisis de soluciones que den lugar a reducir estas y mejorarlas.

\section{GENERACIÓN DE APLICACIÓN TECNOLÓGICA TELEJOB ECUADOR}

En esta etapa se procedió a generar una aplicación dinámica, interactiva y confiable basado en la modalidad de Teletrabajo, con el objetivo que ser el canal de comunicación entre el empleador y el empleado. Esta fue dirigida a la población que accede a la infraestructura tecnológica de los Infocentros en zonas rurales y urbanas marginales. A la aplicación se le denominó "TELEJOB ECUADOR" debido a que representa una forma remota de acceder a ofertas laborales disponibles en la red digital más grande a nivel mundial como lo es el internet. La aplicación TELEJOB ECUADOR genera nuevas oportunidades enmarcadas en la modalidad de teletrabajo, donde la población que accede a los Infocentros Comunitarios a nivel nacional acceda e incursionen en las TIC como una alternativa laboral.

\section{Componentes}

Tanto la página web como la aplicación móvil están desarrolladas con el framework html5: Framework7 además usa la biblioteca Jquery para el manejo del DOM, se decidió utilizar tanto este framework como esa biblioteca ya que proveía componentes visuales compatibles con la visión del proyecto además de proveer métodos compatibles tanto para web como para móvil, lo que facilitó un solo desarrollo para ambas plataformas.

Adicionalmente se utilizó Phonegap para empaquetar el aplicativo web como una aplicación móvil para Android. Para el api se utilizó el lenguaje php, con el framework de desarrollo Laravel 5.6, el cual fue escogido por la sencillez en sus métodos genéricos para el manejo de rutas, permitiendo así crear fácilmente un api rest robusta.

\section{Estructura}

La capa de presentación) está organizada en paquetes y es aquella que una vez implementada será lo que observe el usuario, mientras que la capa de servicios está organizada en espacios de nombres donde contendrá los métodos y procesos que realice la aplicación. La capa de datos se refiere al almacenamiento automático de la información que se vaya ingresando por medio del aplicativo.

\section{RESULTADOS}

A continuación, se detallan los resultados obtenidos en los diferentes puntos de la investigación, los cuales permitieron generar datos, características y estadísticas a fin de evaluar y generar directrices a favor del teletrabajo. Como se detalló en la metodología, para la recolección de 
datos se utilizó la entrevista, en donde se plantearon preguntas de opinión abierta las cuales nos permitieron identificar la importancia del teletrabajo y el impacto que proporciona en los Infocentros comunitarios del país. Se plantearon 10 preguntas a las cuales se les asignó en 3 criterios: criterio alto $=1$ (la opinión es positiva al caso de estudio), medio = 0,5 (no es satisfactorio y presenta contrariedades) y bajo $=0$ (situación difícil con complicaciones) como se aprecia en la Figura 2.

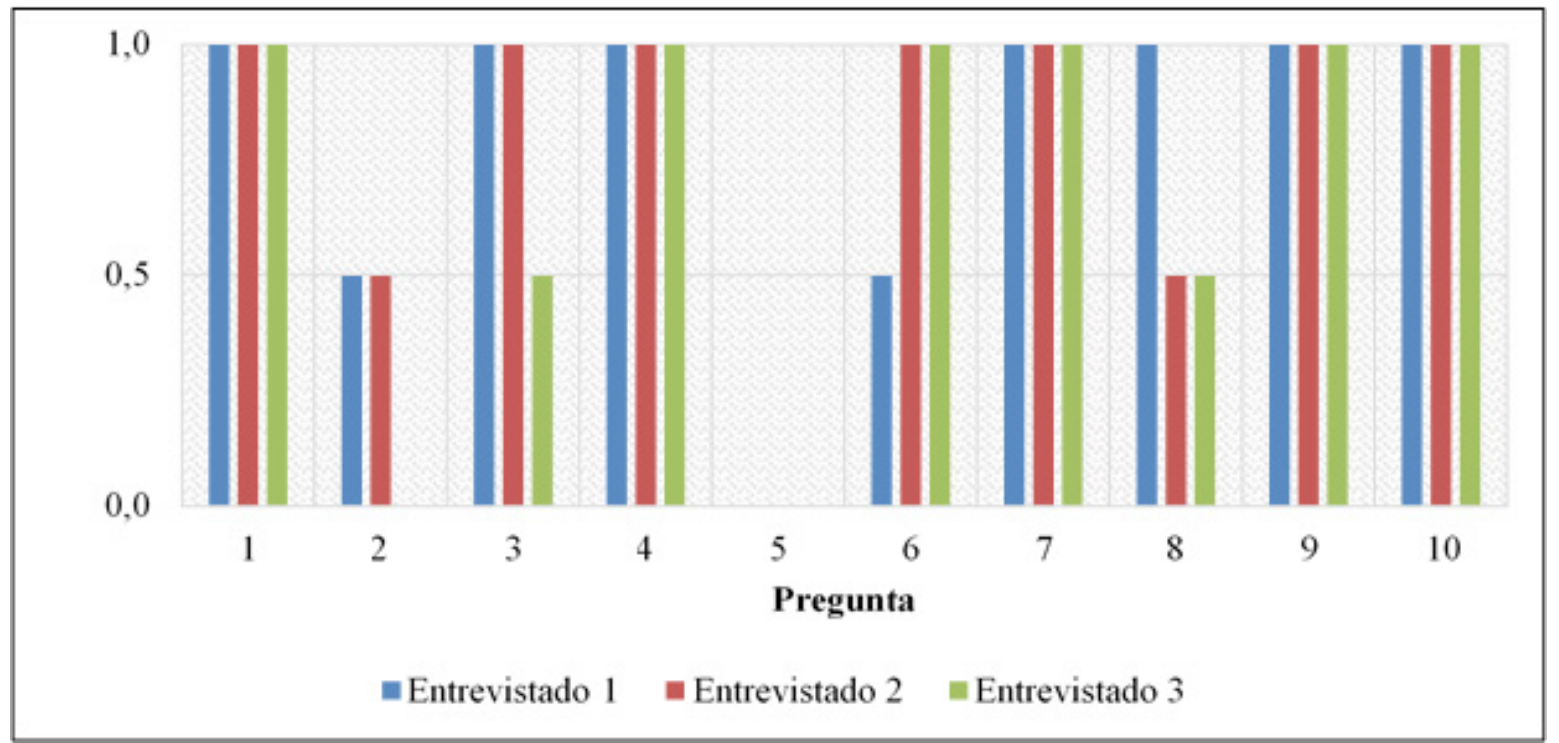

Figura 2. Respuestas obtenidas al cuestionario realizado a personal del MINTEL

A fin de poder analizar la muestra obtenida mediante estadística inferencial, se procedió a reasignar los valores obtenidos ahora entre 0 y 1, dónde 0 representa mínimo o nulo impacto y 1 constituye un medio $u$ alto impacto en la sociedad en lo concerniente al teletrabajo. De esta manera se dilucidaron los valores de impacto medio $(0,5)$ y se procedió a la utilización de una Distribución Binomial $(n=30)$.

De este análisis se obtuvo que el $80 \%$ (Error estándar $=0,13$ ) de respuestas perfilan al teletrabajo como una herramienta con potencial de generar un alto impacto y beneficiar a la sociedad ecuatoriana al ofrecer una menor inversión en infraestructura tecnológica por parte de las empresas, mayor unidad familiar, reducción de gastos asociados a transporte, ahorro de tiempo en desplazamiento e información actualizada de ofertas de trabajo. A pesar de este consenso, para su aplicación se debe tomar las consideraciones y particularidades pertinentes derivadas del análisis cualitativo tales como un posible conflicto de horarios en el uso de los Infocentros, mismos que mantienen una organización y agenda de capacitaciones alineados a una misión y visión estratégicas para este tipo de espacios. A esto se debe sumar la falta de información, publicidad y capacitación en el uso de una aplicación en un computador o en un teléfono móvil.

En la plataforma proporcionada por el MINTEL denominada Observatorio TIC se observa estadísticas de la cantidad, ubicación y porcentaje de cobertura que tiene cada provincia en la implementación de Infocentros Comunitarios (Figura 3). De la misma se desprende que la media de creación de Infocentros en el periodo 2010-2018 es de 95 por año. Las provincias de Chimborazo y Manabí son las que cuentan con un mayor número de Infocentros que corresponden al 8.4 y $7.9 \%$ respectivamente del total a nivel nacional. Por el contrario, Infocentros instalados en 
las provincias del Cañar y Los Ríos representan apenas el 0.93 y $1.87 \%$ respectivamente en el país.

Es importante resaltar que la implementación de Infocentros no es algo rápido de hacer ya que se toma en cuenta varios factores como tipo requerido, cantidad de habitantes o las situaciones en las que se encuentra para poder acceder a uno nuevo.

Se observa además que los Infocentros están centrados en el desarrollo tecnológico y para ello hacen uso de las implementaciones realizadas en cada provincia, permitiendo que las personas se interesen en aprender a manejar un aparato tecnológico como celular y teléfono inteligente, lo cual ha generado beneficios en la sociedad debido a que las diferentes plazas de trabajo requieren personas con aptitudes y conocimientos en computación y con mayor razón mediante la aplicación del proyecto TELEJOB ECUADOR, donde la única manera de acceder a la aplicación es mediante el registro en la aplicación web y su uso mediante la App.

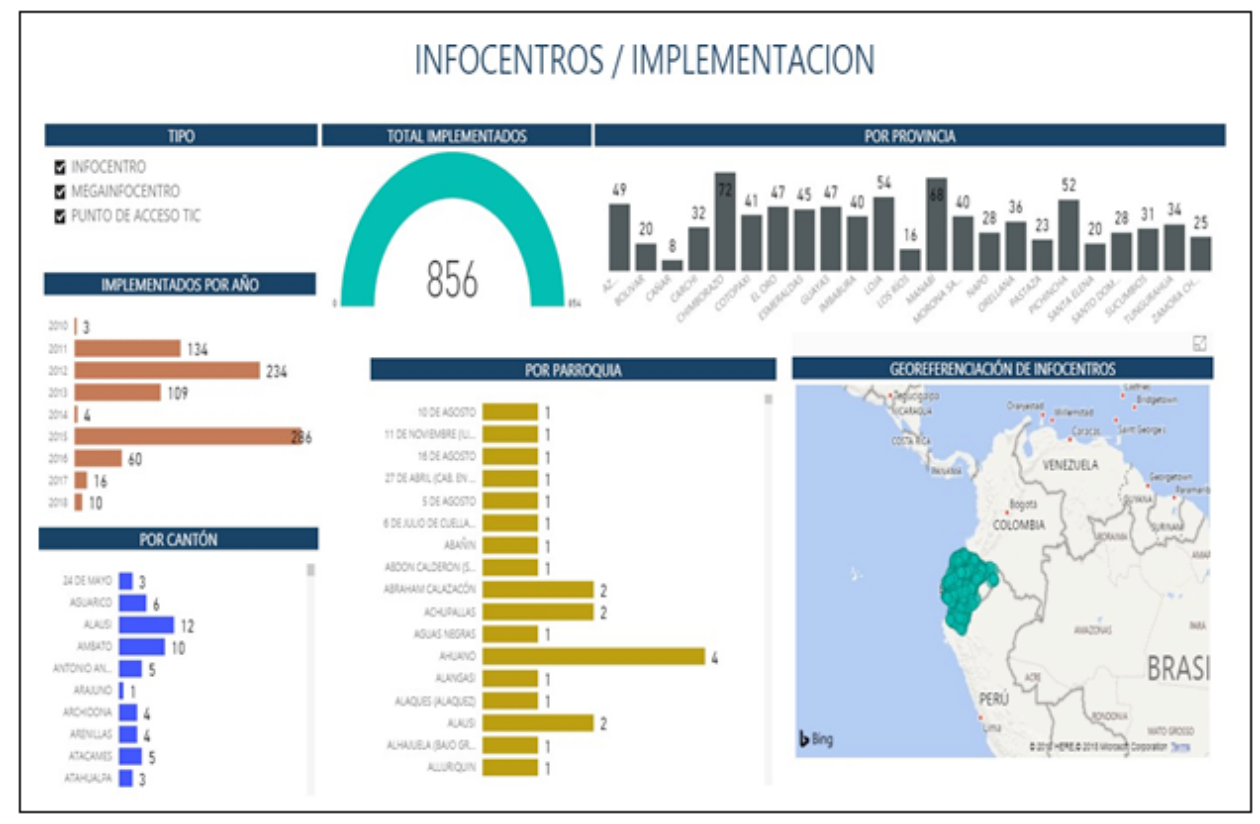

Figura 3. Infocentros en Ecuador. Fuente: ObservatorioTIC- MINTEL

Al recopilar información histórica por el MINTEL presentado en la Figura 4 sobre los avances en infraestructura tecnológica por parte de las empresas en el país, se observa un crecimiento desde el año 2012 al 2015 en todos los campos evaluados. Por ejemplo, la conexión a internet creció un $0.8 \%$, servicio de conexión con banda ancha fija aumentó un $4.3 \%$, uso de redes sociales un $12 \%$, correo electrónico $3.9 \%$ y páginas web empresariales se incrementaron un $18.1 \%$. Muchas de las inversiones realizadas en infraestructura tecnológica son costosas a fin de llegar a mejorar la calidad de servicio y automatizar los procesos que en muchos de los casos requieren tiempo y trabajo adicional del empleado. Sin embargo, para muchas de ellas el costo-beneficio resultará bajo o nulo si la infraestructura implementada no se la usa para generar ingresos. 


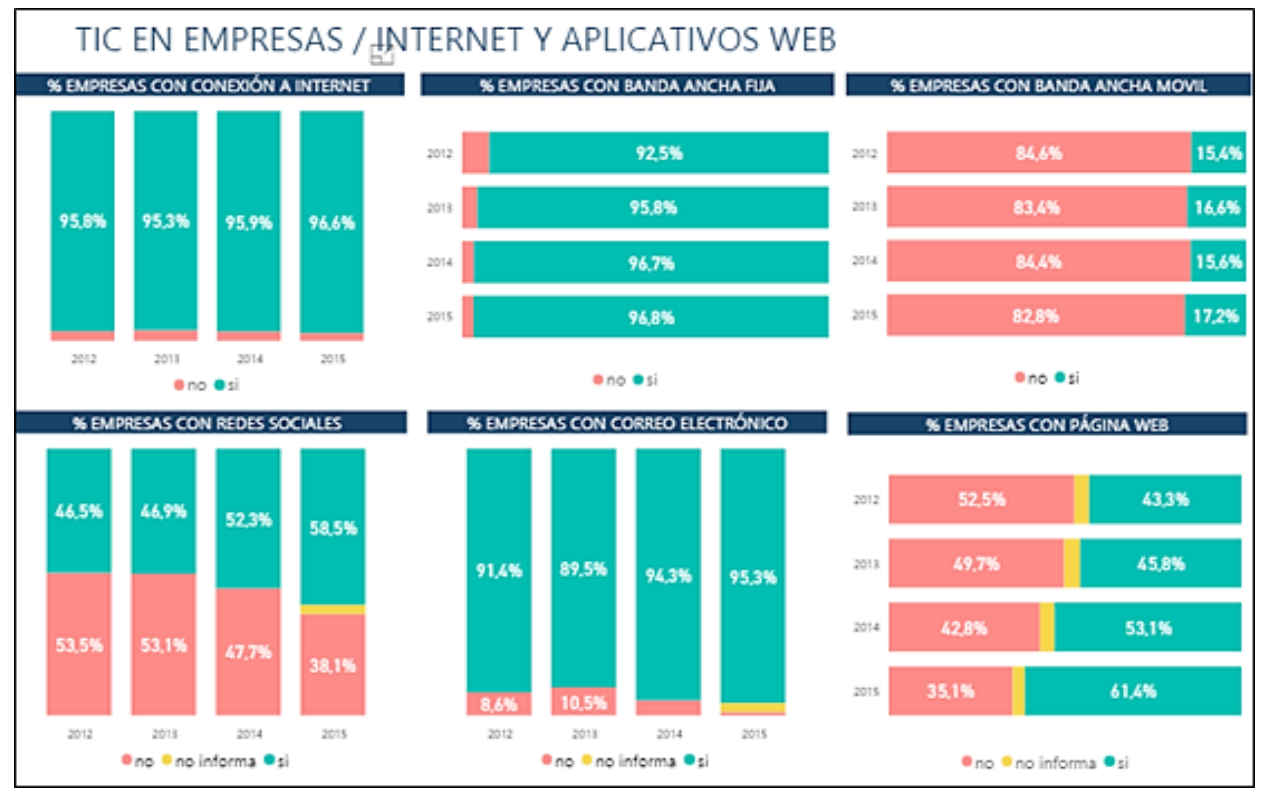

Figura 4. Las empresas vs las Tic. Fuente: Observatorio TIC-MINTEL

En lo concerniente a compras por internet (Figura 5), este tiende a crecer en el país a fin de estar a la vanguardia con los mercados mundiales. La principal actividad de compra se encasilla en el sector comercio con el $33.97 \%$. En ventas, la manufactura de productos lidera con el $29.58 \%$.

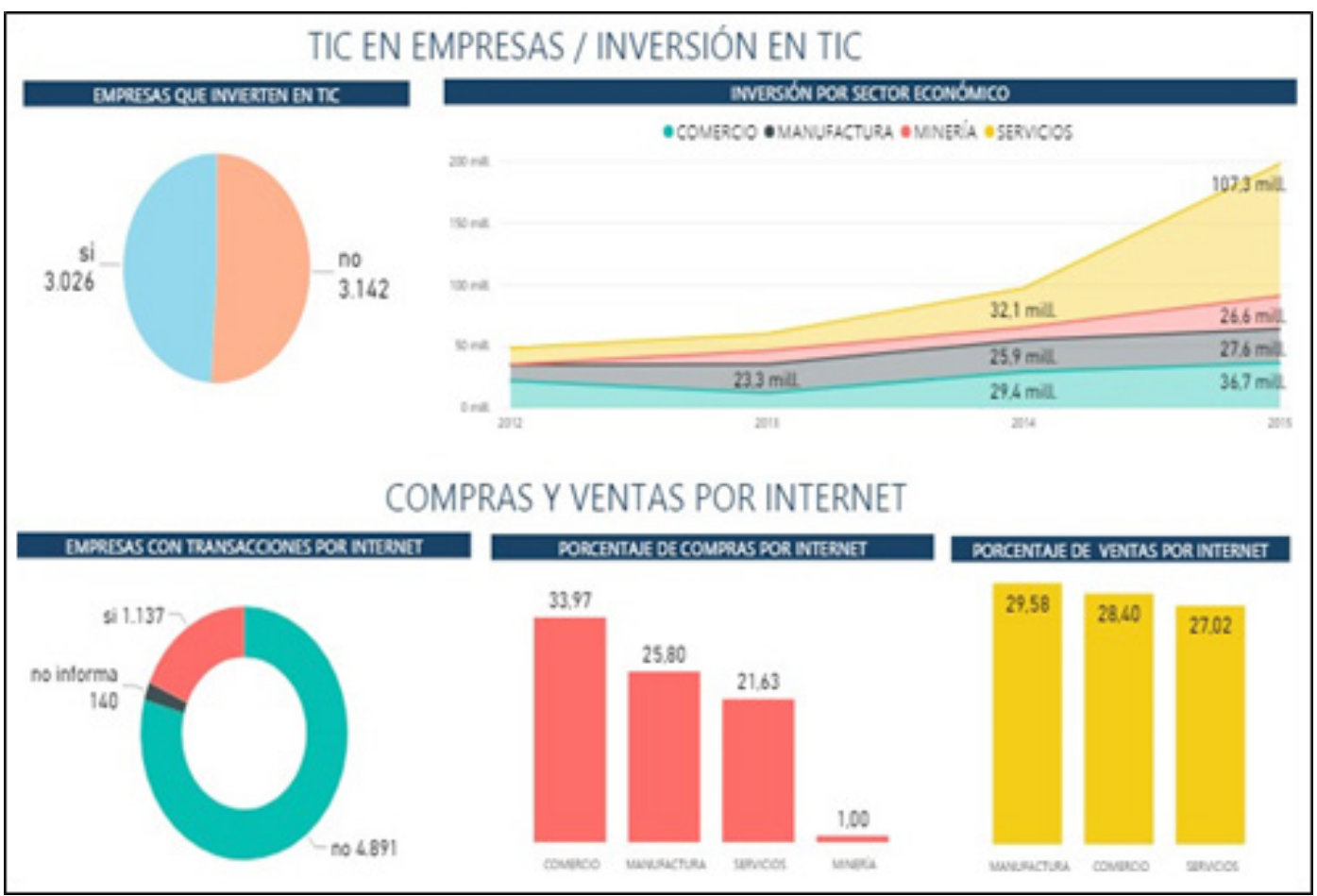

Figura 5. Empresas vs inversiones Tic. Fuente: ObservatorioTIC- MINTEL 
El internet en años atrás era más difícil de obtener en los hogares o en otros sitios lejanos. En la actualidad las empresas que prestan estos servicios han visto la manera de mejorar estos inconvenientes y dar a sus clientes mejor servicio y comodidad. Como se observa en la Figura 6, en el año 2014 las densidades de internet banda ancha fija y móvil en el país eran buenas (37.63\%) pero al compararlas con la gráfica de densidades de la Figura 7, para el año 2018 la misma densidad ha mejorado considerablemente reflejando un crecimiento del $24.64 \%$. Actualmente la mayoría de hogares cuenta con acceso a internet facilitando la conectividad.

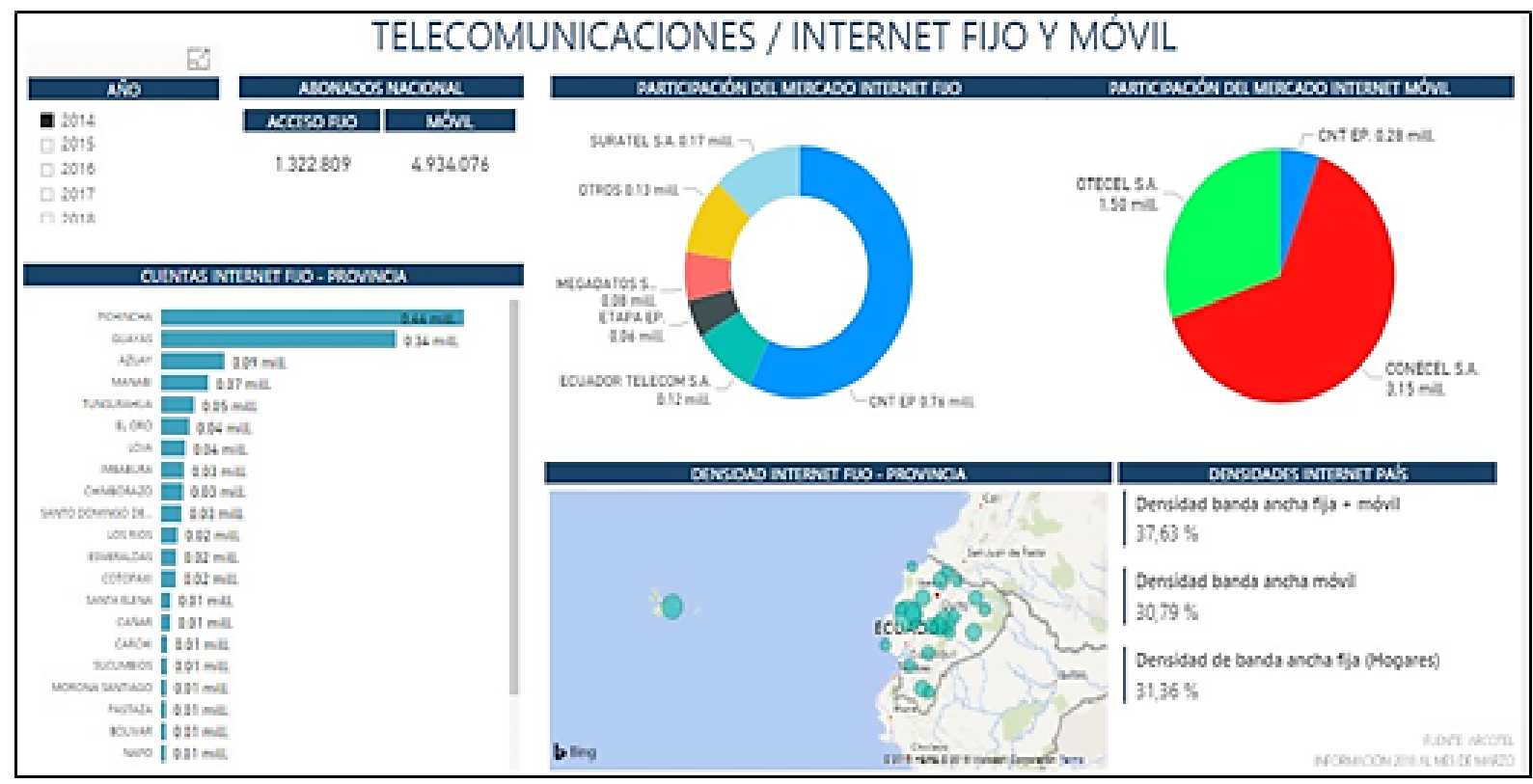

Figura 6. Internet en el 2014. Fuente: Observatorio TIC-MINTEL

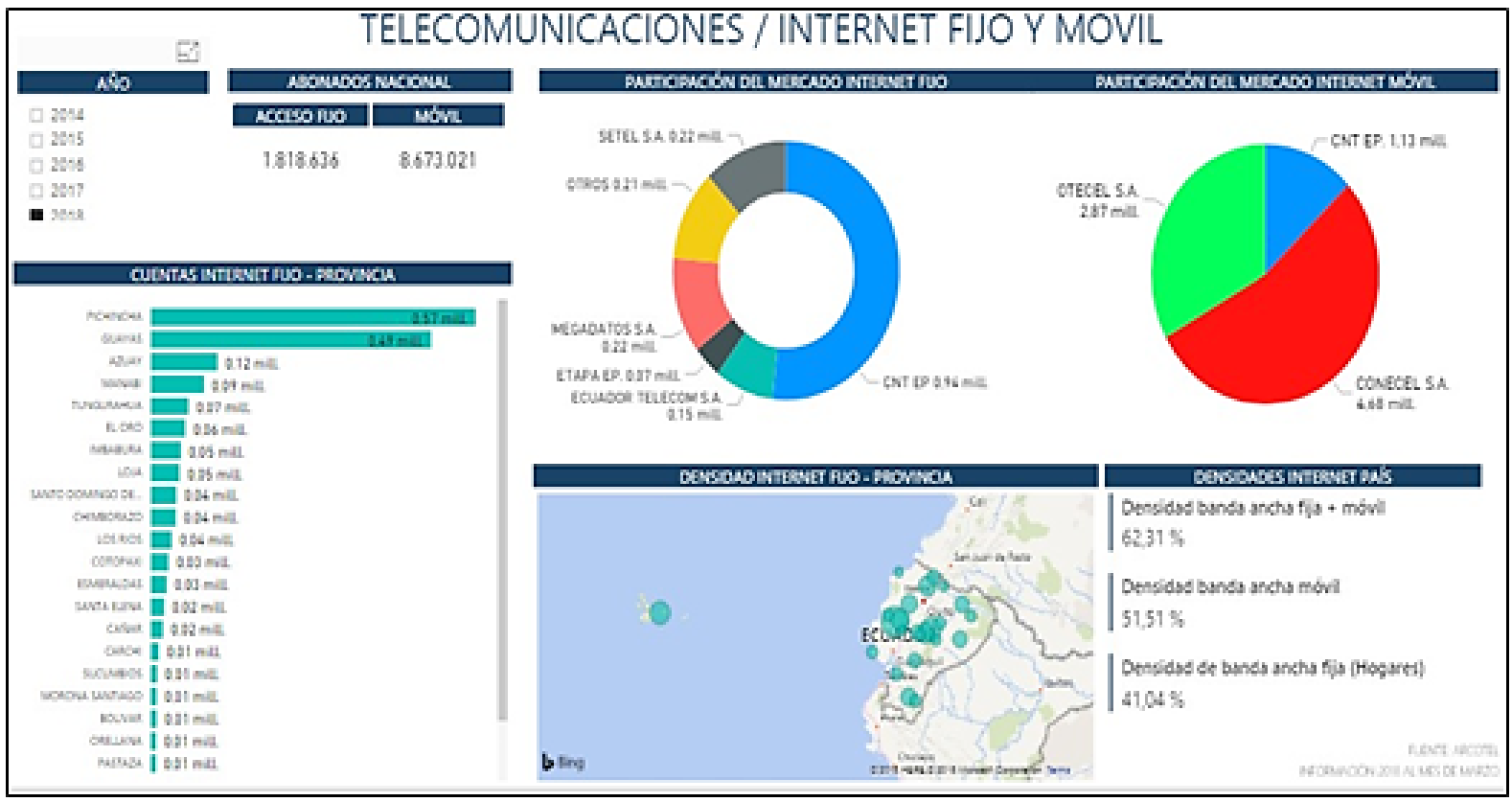

Figura 7. Internet en el 2018. Fuente: ObservatorioTIC- MINTEL 
Finalmente, el uso del internet ha crecido notoriamente desde su primera medición en el año 2009. Se puede observar en la Figura 8 (barras amarillas) que a nivel nacional el mismo ha crecido al 2017 en un $26.66 \%$. Estos datos resultan de gran beneficio ya que implican que más personas de requerirlo podrán acceder a la aplicación que se va implantar y podrán trabajar desde casa sin ningún inconveniente.

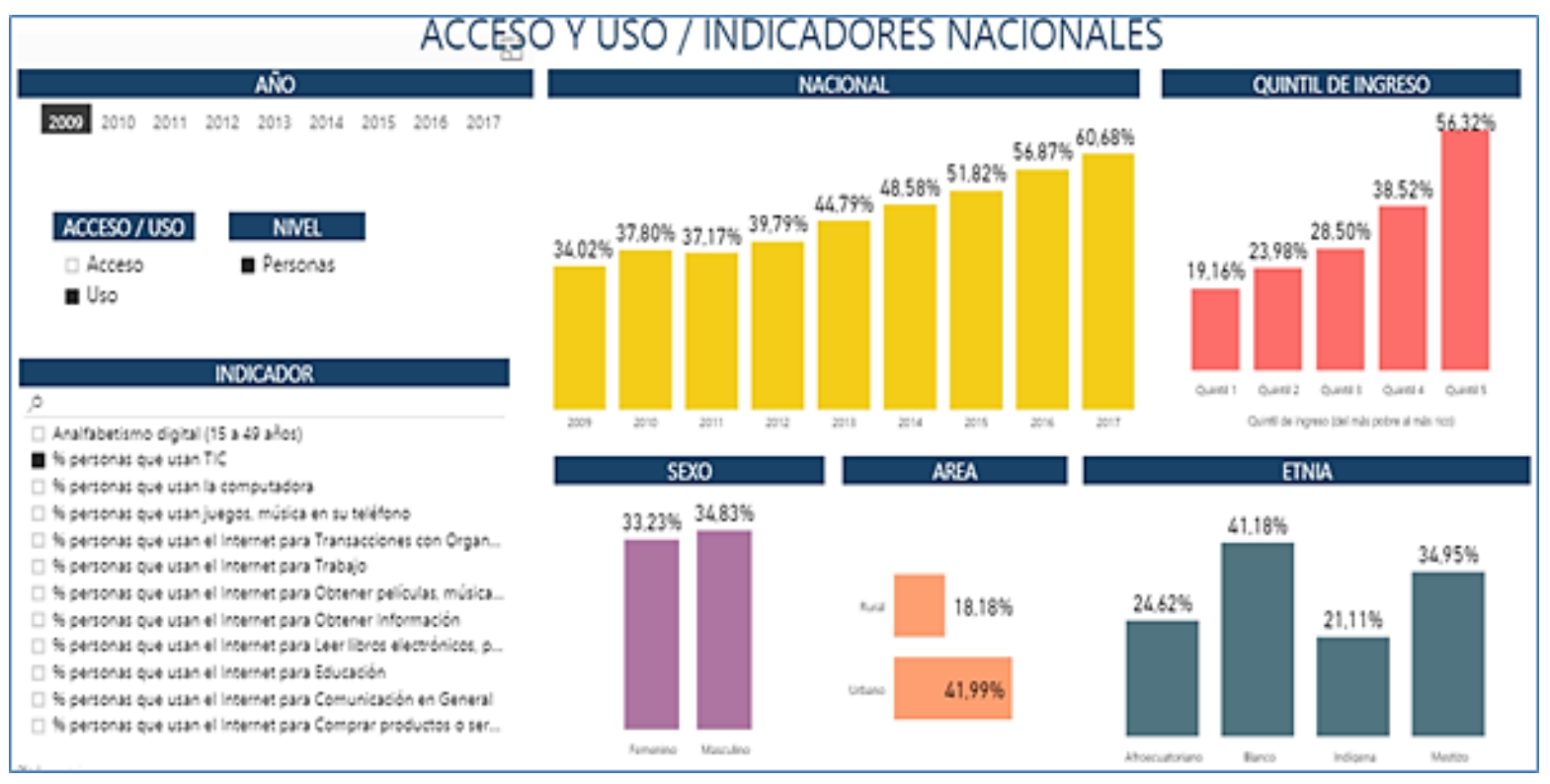

Figura 8. Uso de Internet 2009. Fuente: Observatorio TIC- MINTEL

\section{CONCLUSIONES}

El teletrabajo en Iberoamérica y Latinoamérica empieza a tener un despliegue, Ecuador debe empezar a tomar conciencia de esta realidad y preparar con antelación su infraestructura tecnológica y talento humano. Experiencias positivas como el caso colombiano o el español, a la vanguardia en el tema Teletrabajo, podrían ser adaptadas a la realidad ecuatoriana a fin de llegar al objetivo de proporcionar ganancias rentables a las empresas y a los trabajadores reduciendo distancias y tiempo de entrega de resultados a tareas asignadas.

El éxito de la aplicación informática para teletrabajo denominada, TELEJOB ECUADOR radica en el acceso y enrolamiento de la población en las tecnologías de la información y comunicaciones, donde los usuarios acceden y revisan diferentes ofertas laborales, con la finalidad de postularse a la que mejor se apegue a su perfil, mientras que el usuario contratante analiza los perfiles de los postulados para elegir al más idóneo.

En respuesta a este factor clave en el Teletrabajo, se desarrolló la aplicación TELEJOB ECUADOR, misma que canaliza el desconocimiento de ambas partes sobre Teletrabajo y guía de forma ordenada el proceso para su aplicación con el objetivo de reducir y ser el canal de comunicación entre un empleador y el ciudadano en búsqueda laboral disminuyendo la incertidumbre en su operatividad, remuneración, seguridad y rentabilidad. 
La implementación en la infraestructura tecnológica de la Red de Infocentros creados por el MINTEL es factible realizarla. A fin de evitar conflictos con las actividades ya programadas en estos centros se plantea que personas que están alejadas de las ciudades pueden hacer uso de los Infocentros en ciertos horarios para poder acceder a la aplicación y usarla buscando empleos eventuales y desarrollándolos en el caso que se necesite un computador. Caso contrario los Infocentros serán únicamente el punto de encuentro entre el usuario y la aplicación que proporciona ofertas de trabajo. Con esta dinámica se cumple y mantiene la misión para la cual los Infocentros fueron creados de impartir conocimientos y brindar oportunidades a las poblaciones menos favorecidas y vulnerables.

Adicionalmente la creación de esta aplicación es favorable porque es un tema y una modalidad de trabajo a emplear en cualquier parte del país, por lo que los ciudadanos pueden acceder a la misma no necesariamente en los Infocentros, sino que en caso de contar con un dispositivo móvil podrían trabajar desde ahí. 


\section{REFERENCIAS BIBLIOGRÁFICAS}

Bécares, B. (2014). silicoWeek. Obtenido de Teletrabajo: ¿es algo tan bueno como nos cuentan?: https://www. siliconweek.com/cloud/teletrabajo-es- tan-bueno-como-nos-cuentan-54414

Bonilla, L., Plaza, D., Soacha, G., \& Riaño, M. (2014). Teletrabajo y su Relación con la Seguridad y Salud en el Trabajo. Ciencia \& Trabajo, 38-42. Obtenido de Teletrabajo y su relación con la seguridad y salud en el trabajo: http://www.cienciaytrabajo.cl/cytqa/Paginas/Teletrab ajo-y-su-Relaci\%C3\%B3n-con-la-Seguridad-y- Salud-en-el-Trabajo.aspx

Civit, C., \& March, M. (2000). Implantación del teletrabajo en la empresa. Barcelona: Gestión 2000.

Ericsson ConsumerLab Insight Summary Report. (2015). Flexibility in Work Life. Stockholm: Ericsson.

Havriluk, O. (2010). El Teletrabajo: Una opción en la era digital. Observatorio Laboral Revista Venezolana, 93109.

INEC, I. N. (2016). Tecnologías de la Información y Comunicación-TIC. Obtenido de http://www.ecuadorencifras. gob.ec/documentos/web-inec/Estadisticas_Sociales/TIC/2016/170125.Present acion_Tics_2016.pdf

López, N. (2017). Propuesta de Marco de Referencia para Teletrabajo en Ecuador y soporte mediante Tecnologías de Información, Caso de estudio Empresa ATIJAGUAR CÍA. LTDA. (Tesis). Quito: Universidad de las Américas.

Ministerio del Trabajo. (2016). Teletrabajo. ACUERDO MINISTERIAL No. MDT-2016-190.

Ministerio Relaciones Laborales. (Enero de 2018). Ministerio del Trabajo. Recuperado el 17 de Mayo de 2018, de Ministerio del Trabajo: www.trabajo.gob.ec/relanzamiento-de-teletrabajo- en-el-ecuador/

MINTEL. (Abril de 2017). PROYECTO: KO04 MINTEL - Ampliación de la Red Infocentros. Obtenido de Ministerio de Telecomunicaciones y de la Sociedad de la Información: https://www.telecomunicaciones.gob.ec/wpcontent/uploads/2017/04/AMPLIACION-DE-LA- RED-INFOCENTROS.pdf

MINTEL. (2018). Infocentros Comunitarios: una oportunidad para crecer. Obtenido de https://observatoriotic. mintel.gob.ec/infocentros- comunitarios-una-oportunidad-para-crecer/

Sánchez, M. (2012). Un acercamiento a la medición del teletrabajo: evidencia de algunos países de América Latina. Santiago: Comisión Económica para América Latina y el Caribe.

Ushakova, T. (2015). El Derecho de la OIT para el trabajo a distancia: ¿una regulación superada o todavía aplicable? Revista Internacional y Comparada de RELACIONES LABORALES Y DERECHO DEL EMPLEO, $1-19$. 